\title{
Dehydration Kinetics of Sibutramine Hydrochloride Monohydrate $\uparrow$
}

\author{
A. RAVIKIRAN*, M. ARTHANAREESWARI, \\ P. KAMARAJ, CH. PRAVEEN and K.V PAVAN
}

Department of Chemistry, SRM University, SRM Nagar, Kattankulathur-603203, Kancheepuram District, Tamilnadu, India

ravianalytical@gmail.com

Received 8 January 2013 / Accepted 15 February 2013

\begin{abstract}
Sibutramine hydrochloride ( $\mathrm{SH}$ ) is used for the treatment of obesity. The comprehensive physical characterization of drug and drug product is gaining importance due to the potential impacts of phase transitions on the drug product's quality and performance. In this work, the kinetics of dehydration of SH-monohydrate were studied using thermogravimetry-differential thermal analysis (TG-DTA) and the solid-state characterization was done by using differential scanning calorimetry (DSC), powder x-ray diffraction (PXRD) and scanning electron microscopy (SEM). The weight loss data obtained from thermogravimetry (TG) was used for non-isothermal kinetics analysis and the well accepted Coats-Redfern model fitting approach was applied to determine kinetic parameters. Total thirteen solid state reaction models were evaluated, diffusion control (Crank) model found to be the best fit model for this reaction with an excellent correlation 0.9984. The values of Arrhenius parameters, activation energy $\left(E_{a}\right)$ and pre-exponential factor $(A)$ were found to be $34 \mathrm{kcal} / \mathrm{mol}$ and $3.23 \times 10^{16} \mathrm{sec}^{-1}$ respectively. The dehydration of $\mathrm{SH}$ Monohydrate resulted anhydrous $\mathrm{SH}$ with a distinct $\mathrm{x}$-ray diffraction profile and crystal morphology.
\end{abstract}

Keywords: Dehydration, Sibutramine hydrochloride, Model fitting, Kinetics, PXRD, TG, DSC

\section{Introduction}

Active pharmaceutical ingredients (APIs) are known to exist in different solid forms, namely polymorphs, hydrate/solvates and amorphous forms. About one third of APIs are able to form hydrates, the molecular adducts of API with water ${ }^{1,2}$. Operational conditions and manufacturing process may change the hydration state of an API, which might impact dissolution rate, stability, drug assay and bioavailability ${ }^{3}$. In view of increasing regulatory concern related to solid form of the drug substance, it is highly important to understand the physical stability and inter-convertibility of the drug solid forms ${ }^{4-6}$. Therefore, through understanding of the kinetics of solid state reactions of drug substance leaves an opportunity to prevent and to have control over the consequent physical changes, which may occur during manufacturing process and shelf life of drug product.

$\uparrow$ Presented to the National Conference on Chemistry Solutions at SRM University, India 


\section{Experimental}

Mettler Toledo TGA/SDTA (Model $851^{\mathrm{e}}$ ) with STARe software was used for recording and processing TG-DTA thermogram of $\mathrm{SH}$ monohydrate. Accurately weighed powder of $15.8240 \mathrm{mg}$ was taken into an aluminum pan $(100 \mu \mathrm{L})$, heated at a programmed rate of $5{ }^{\circ} \mathrm{C} / \mathrm{min}$ in the temperature range from $25^{\circ}$ to $150{ }^{\circ} \mathrm{C}$ under nitrogen flow of $50 \mathrm{~mL} / \mathrm{min}$.

In the present work, the dehydration of sibutramine hydrochloride $(\mathrm{SH})$ monohydrate, chemically, is a racemic mixture of the $(+)$ and (-) enantiomers of cyclobutanemethanamine, 1-(4-chlorophenyl)- $N, N$-dimethyl- $\alpha$-(2-methylpropyl)-, hydrochloride, monohydrate, represented in Figure 1, was studied by thermogravimetry-differential thermal analysis (TG-DTA). API of SH is used for the treatment of obesity. Studies connected to crystal chemistry and decomposition kinetics of SH-monohydrate have been reported ${ }^{7,8}$, but a thorough review revealed that there were no attempts made in relation to the study of dehydration kinetics of SH monohydrate in the published literature.

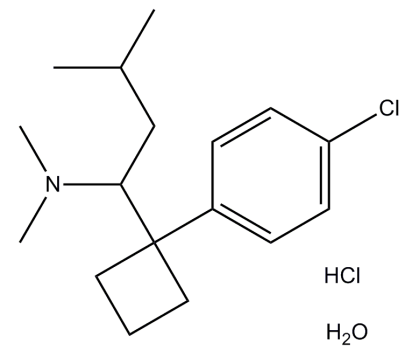

Figure 1. Molecular structure of SH-monohydrate

TG is often used to study the kinetics of solid state reactions involving weight change during reaction ${ }^{9,10}$. Some examples of such reactions include thermal decomposition, oxidation and dehydration or desolvation. In this work model fitting approach was applied on the collected weight loss data using Coats-Redfern method ${ }^{11,12}$. Physical characterization of SH-monohydrate and $\mathrm{SH}$-anhydrous was done using differential scanning calorimetry (DSC), powder x-ray diffraction (PXRD) and scanning electron microscope (SEM).

\section{Mathematical}

General form of rate equation of solid state reactions used for isothermal analysis is

$$
\frac{d x}{d y}=k(T) f(\alpha)
$$

Where $\alpha$ is the extent of reaction, $t$ is the time, $T$ is the temperature and $f(\alpha)$ is reaction model. For the current dehydration study:

$$
\alpha=\frac{w_{o}-w_{t}}{B_{o} w_{o}}
$$

Where $w_{o}, w_{t}$ and $B$ are the initial mass of the sample, the mass at time $t$ and the fraction of weight loss for complete dehydration of the reaction, respectively. Integral form of above equation (1) is

$$
g(\alpha)=k t, \text { with } g(\alpha)=\int_{0}^{\alpha} \frac{1}{f(\alpha)} d \alpha
$$


According to Arrhenius equation

$$
k=A e \frac{-E a}{R T}
$$

Where $k, E_{a}$ and $A$ are the rate constant, the activation energy and the pre-exponential factor, respectively.

$$
g(\alpha)=A e \frac{-E a}{R T} t
$$

Under non-isothermal conditions integral form of the above equation is

$$
g(\alpha)=\frac{A}{\beta} \int_{0}^{T} e \frac{-E a}{R T} d t
$$

Where $\beta$ is heating rate

If $\frac{E a}{R T}$ is replaced by a variable $x$, the above temperature integral equation (6) becomes

$$
g(\alpha)=\frac{A E a}{B R} \int_{x}^{\infty} \frac{e^{-x}}{x^{2}} d x
$$

If $p(x)=\int_{x}^{\infty} \frac{e^{-x}}{x^{2}}$, then above equation (7) becomes

$$
g(\alpha)=\frac{A E a}{\beta R} p(x)
$$

Where, $p(x)$ is the exponential integral, it has no analytical solutions but has many approximations ${ }^{13}$. The above equation can be used for several model fitting methods for the analysis of non-isothermal kinetics. Model-fitting approach involves fitting different models to $\alpha$-temperature curves. In this study popular Coats-Redfern method was used in which the asymptotic series expansion for approximating the above equation 8 is used to get the following equation:

$$
\ln \frac{g(\alpha)}{T^{2}}=\ln \left[\frac{A R}{\beta E a}\left(1-\frac{2 R T}{E a}\right)\right]-\frac{E a}{R T}
$$

Where, $T$ is the mean experimental temperature. The values of $E_{a}$ and $A$ can be obtained from slope and Intercept values respectively from the graph plotted for $\ln \frac{g(\alpha)}{T^{2}}$ versus $1 / T$ for different models.

\section{Powder $x$-ray diffraction (PXRD)}

Powder $\mathrm{x}$-ray diffractograms of $\mathrm{SH}$ monohydrate and $\mathrm{SH}$ anhydrous (Obtained after dehydration of SH monohydrate) were collected on Bruker Powder x-ray diffractometer (Model D8 ADVANCE). Peak position accuracy and relative intensity was checked using NIST standard reference material 1976a (Corundum). The x-ray tube was operated at a voltage of $40 \mathrm{kV}$ and current of $40 \mathrm{~mA}$. Each diffraction profile was collected in continuous mode and in the scan range of $3^{\circ}$ to $45^{\circ} 2 \theta$ with a step size of $0.01^{\circ} 2 \theta$ and with a time per step of $0.1 \mathrm{sec}$. PMMA (Poly-methyl methacrylate) sample holder (Ø $25 \mathrm{~mm}$ ) was used for the specimen preparation. Top loading method was followed. Data acquisition and analysis were performed on Bruker's XRD commander and Eva software respectively. 


\section{Differential scanning calorimetry (DSC)}

Mettler Toledo DSC (Model-821 ${ }^{\mathrm{e}}$ ) with STARe software was used for recording and processing DSC thermograms of SH monohydrate. Accurately weighed powder was taken into a closed aluminum pan $(40 \mu \mathrm{L})$, heated at a programmed rate of $10{ }^{\circ} \mathrm{C} / \mathrm{min}$ in the temperature range from $25^{\circ}$ to $220{ }^{\circ} \mathrm{C}$ under nitrogen flow of $40 \mathrm{~mL} / \mathrm{min}$. Empty aluminum pan was used as a reference.

\section{Scanning electron microscopy (SEM)}

The SEM images of SH monohydrate and SH anhydrous (Obtained from dehydration of SH -monohydrate) were collected using JEOL scanning electron microscope (Model JSM6380). The powders were mounted onto aluminum stubs with the help of double sided adhesive carbon tape (Nissin EM Co. Ltd., Tokyo) then the powders were sputter coated with Platinum under vacuum by using JEOL auto fine coater (Model JFC-1600). The specimens were directly observed under microscope at a magnification of $500 \mathrm{X}$.

\section{Results and Discussion}

\section{Thermogravimetry-differential thermal analysis (TG-DTA)}

The TG-DTA thermogram was represented in Figure 2. The weight loss due to dehydration was observed till about $95{ }^{\circ} \mathrm{C}$, the weight loss value found to be about $5.4649 \% \mathrm{w} / \mathrm{w}$. The data was subjected to model fitting kinetics analysis.

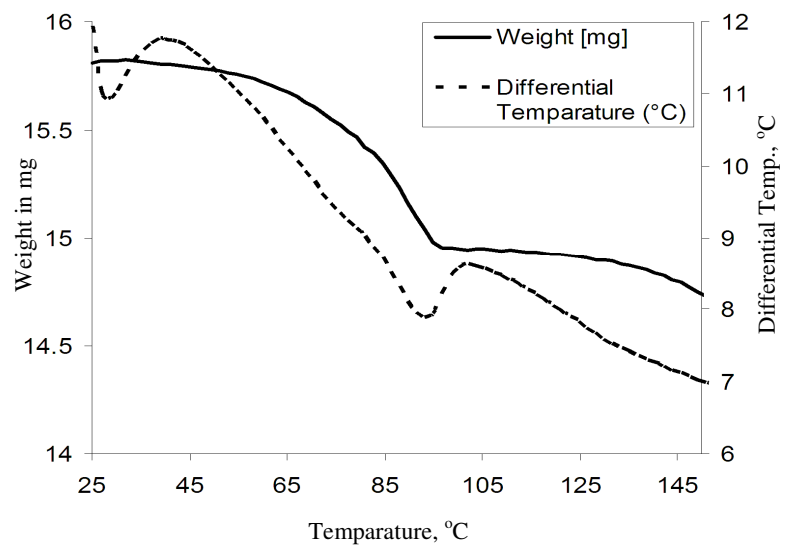

Figure 2. Overlaid thermogram of TG (-) and DTA (----) graphs of SH-monohydrate.

\section{Kinetics analysis of $T G$ data}

Using various expressions for $\mathrm{g}(\alpha)$ in equation 9 Arrhenius parameters were calculated from the plot of $\ln \frac{g(\alpha)}{T^{2}}$ against $1 / T$ for $0 \leq \alpha \leq 1$. The combined graphs of all thirteen models were represented in Figure 3. The set of calculated Arrhenius parameters for dehydration of SH monohydrate are listed in Table 1. For each model, goodness fit was determined by the correlation value, represented in Table 1. The first six reaction models have very small values of pre-exponential factor demonstrating that these models cannot explain the reaction kinetics. Among the remaining models, diffusion control (Crank) model has got maximum correlation of 0.9984 . Thus this model could explain the dehydration reaction of $\mathrm{SH}-$ monohydrate and the calculated activation energy and pre-exponential factor for this model were $34 \mathrm{kcal} / \mathrm{mol}$ and $3.23 \times 10^{16} \mathrm{sec}^{-1}$ respectively. 
Table 1. The evaluated solid state reaction models and corresponding calculated values of Arrhenius parameters for the dehydration kinetics of sibutramine hydrochloride monohydrate

\begin{tabular}{cccccc}
\hline Reaction Model & $\mathrm{f}(\alpha)$ & $\mathrm{g}(\alpha)$ & $\begin{array}{c}\mathrm{Ea} \\
\mathrm{kcal} / \mathrm{mol}\end{array}$ & $\begin{array}{c}\mathrm{A} \\
\mathrm{sec}^{-1}\end{array}$ & $-\mathrm{R}$ \\
\hline Power Law & $4 \alpha^{3 / 4}$ & Nucleation models & & & \\
Power Law & $3 \alpha^{2 / 3}$ & $\alpha^{1 / 4}$ & 2 & 0.01 & 0.9957 \\
Power Law & $2 \alpha^{1 / 2}$ & $\alpha^{1 / 3}$ & 4 & 0.03 & 0.9968 \\
Avrami-Erofeev & $\begin{array}{c}4(1-\alpha) \\
\alpha^{1 / 2}\end{array}$ & 1 & 0.00 & 0.9739 \\
Avrami-Erofeev & $\begin{array}{c}3(1-\alpha)]^{3 / 4} \\
{[-\ln (1-\alpha)]^{2 / 3}}\end{array}$ & {$[-\ln (1-\alpha)]^{1 / 4}$} & 4 & 0.06 & 0.9820 \\
Avrami-Erofeev & $2(1-\alpha)$ & {$[-\ln (1-\alpha)]^{1 / 3}$} & 6 & 0.91 & 0.9846 \\
& {$[-\ln (1-\alpha)]^{1 / 2}$} & {$[-\ln (1-\alpha)]^{1 / 2}$} & 9 & $1.95 \times 10^{2}$ & 0.9866
\end{tabular}

One dimensional

Diffusion

Diffusion models

Diffusion control

(Janders)
Diffusion control

$1 / 2 \alpha^{-1}$

$\alpha^{2}$

29

$2.46 \times 10^{14} \quad 0.9983$

$$
\begin{gathered}
2(1-\alpha)^{2 / 3} \\
{\left[1-(1-\alpha)^{1 / 3}\right]^{-1}}
\end{gathered}
$$

$\left[1-(1-\alpha)^{1 / 3}\right]^{2}$

37

$2.47 \times 10^{18} \quad 0.9959$

(Crank)

$$
3 / 2\left[(1-\alpha)^{-1 / 3}-1\right]^{-1} \quad 1-2 / 3 \square \alpha-(1-
$$

34

$3.23 \times 10^{16} \quad 0.9984$

Reaction order and geometrical contraction models

Mampel

(first order)

Second Order

Contracting

cylinder

Contracting

Sphere

$\begin{array}{ccccc}1-\alpha & {[-\ln (1-\alpha)]} & 20 & 1.24 \times 10^{9} & 0.9883 \\ (1-\alpha)^{2} & (1-\alpha)^{-1}-1 & 29 & 9.73 \times 10^{14} & 0.9478 \\ 2(1-\alpha)^{1 / 2} & 1-(1-\alpha)^{1 / 2} & 17 & 3.42 \times 10^{6} & 0.9977 \\ 3(1-\alpha)^{2 / 3} & 1-(1-\alpha)^{1 / 3} & 18 & 1.14 \times 10^{7} & 0.9956\end{array}$

$\rightarrow$ Model-1

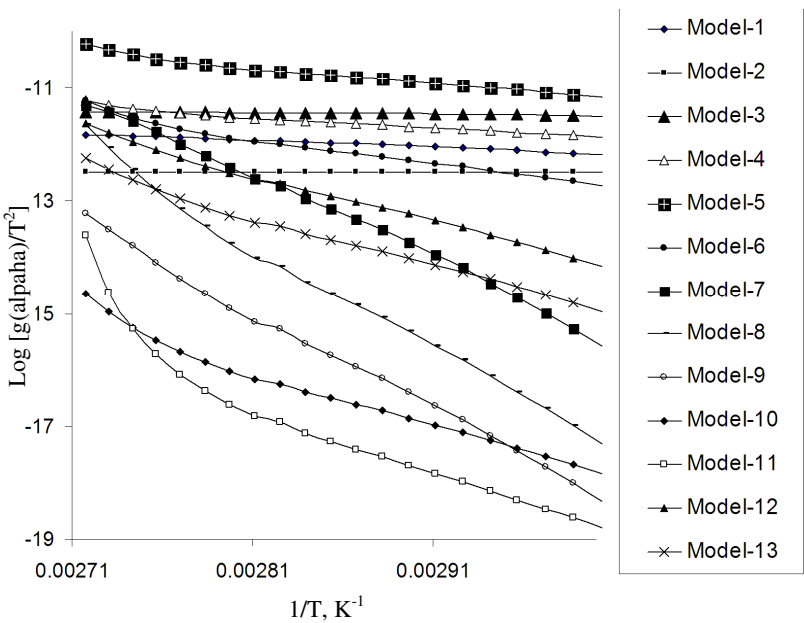

$1 / \mathrm{T}, \mathrm{K}^{-1}$

Figure 3. A combined graph representing total thirteen solid-state reaction models, evaluated according to the equaton-9 


\section{Powder $x$-ray diffraction $(P X R D)$}

An overlay of powder x-ray diffractograms of $\mathrm{SH}$ monohydrate and $\mathrm{SH}$ anhydrous (Obtained from dehydration of SH monohydrate) was represented in Figure 4. The data indicates occurrence of different crystalline phase after dehydration.

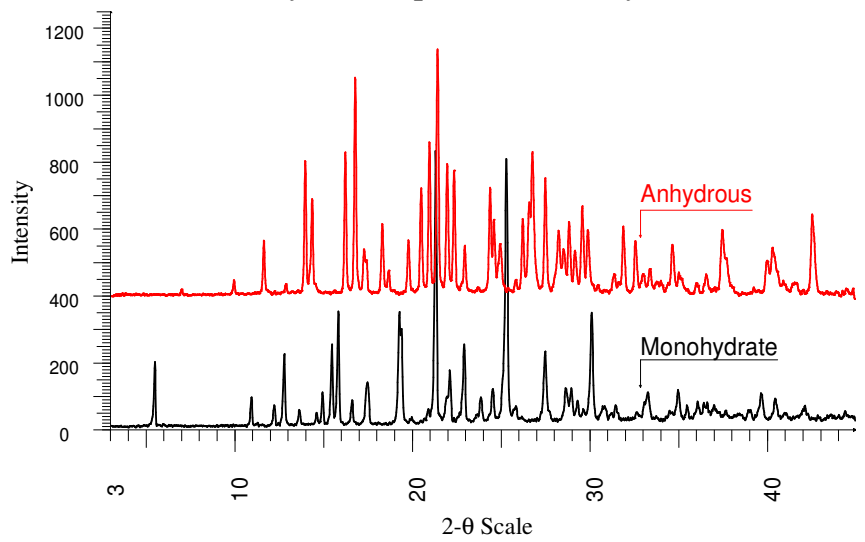

Figure 4. Overlay of PXRD profiles of SH-monohydrate and Anhydrous obtained from dehydration of SH-monohydrate

\section{Differential scanning calorimetry (DSC)}

The DSC thermogram represents a dehydration endotherm of SH-monohydrate at about $100{ }^{\circ} \mathrm{C}$ followed by a melting endotherm of $\mathrm{SH}$-anhydrous form at about $195{ }^{\circ} \mathrm{C}$. The DSC thermogram was represented in Figure 5.

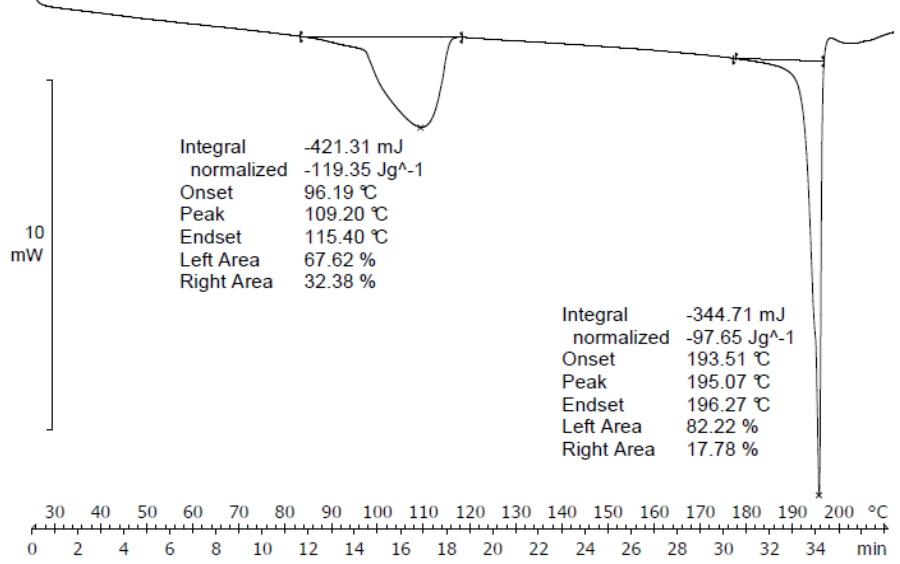

Figure 5. DSC thermogram of SH-monohydrate: Dehydration endotherm followed by melting endotherm

\section{Scanning electron microscopy (SEM)}

Morphological changes associated with increase in crystallite size were evident from SEM images of SH-Monohydrate powder before and after dehydration. Figure 6 represents the SEM images of SH Monohydrate and SH anhydrous (Obtained from dehydration of SH monohydrate). 

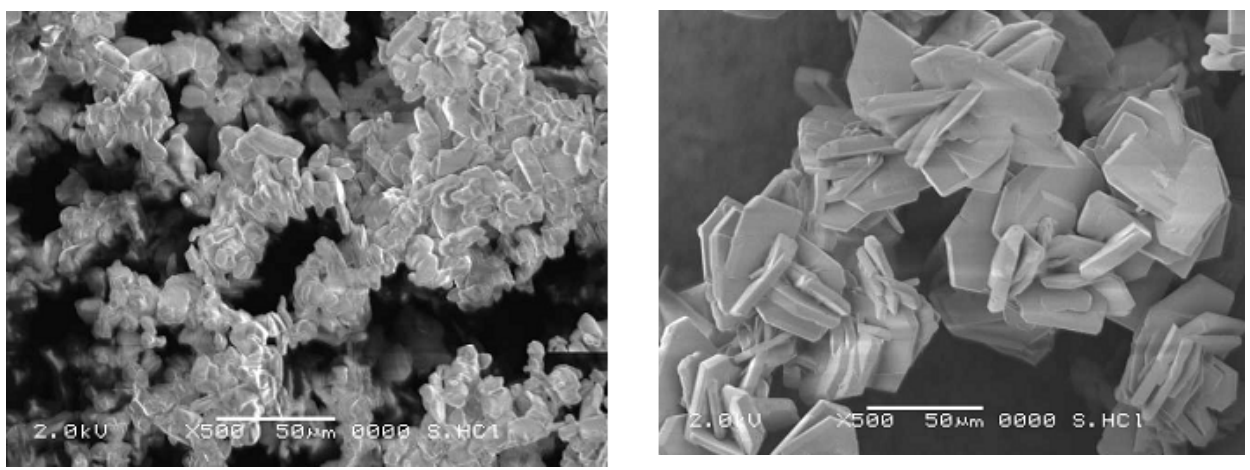

Figure 6. SEM images of SH-monohydrate (left) and SH-anhydrous (right)

\section{Conclusion}

Thermal analysis (TG-DTA and DSC) of sibutramine hydrochloride monohydrate is used to understand dehydration kinetics and subsequent changes. Non-isothermal kinetics analysis was successfully performed on TG data for $0 \leq \alpha \leq 1$, i.e. till $95{ }^{\circ} \mathrm{C}$, where the dehydration reaction was completed. The dehydration of $\mathrm{SH}$-monohydrate could best be explained by diffusion control (Crank) model. The calculated activation energy $(E a)$ and pre-exponential factor (A) values for that model were found to be $34 \mathrm{k} . \mathrm{cal} / \mathrm{mol}$ and $3.23 \times 10^{16} \mathrm{sec}^{-1}$, respectively. Dehydration of SH-monohydrate resulted in morphological changes associated with increase in particle size and the crystallographic changes were confirmed by using PXRD analysis.

\section{References}

1. Vippagunta S R, Brittain H G and Grant D J W, Adv Drug Deliver Rev., 2001, 48, 3-26.

2. Stahl H P, Towards Better Safety Drugs and Pharmaceutical Products; Braimer, D. D., Ed.; Biomedical Press; Elsevier North Holland: Amsterdam, 1980; 265-280

3. Khankari R K and Grant D J W, Thermochim Acta, 1995, 248, 61-79.

4. $\quad$ Elder J P, Thermochim Acta, 1994, 234, 153-164.

5. Raw A S, Furness M S, Gill D S, Adams R C, Holcombe F O Jr and Yu L X, Adv Drug Deliv Rev., 2004, 56(3), 397-414.

6. Yu L X, Furness M S, Raw A, Outlaw K P W, Nashed N E, Ramos E, Miller S P F, Richard C A, Florence F, Rashmikant M P, Jr Frank O H, Yuan-Y C and Ajaz S H, Pharm Res., 2003, 20(4), 531-536.

7. Oliveira P R, Stulzer H K, Bernardi L S, Borgmann S H M, Cardoso S G and Silva M A S, J Therm Anal Cal., 2010, 100, 277-282.

8. Maccaroni E, Alberti E, Malpezzi L, Masciocchi N and Pellegatta C, J Pharm Sci., 2008, 97, 5229-5239.

9. Tanaka H, Thermochim Acta, 1995, 267(1), 29-44.

10. MacCallum J R and Tanner J, Eur Polym J., 1970, 6(7), 1033-1039.

11. Vyazovkin S and Wight C A, Thermochim Acta, 1999, 340, 53-68.

12. Coats A W and Redfern J P, Nature, 1964, 201, 68-69.

13. Doyle C D, Nature, 1965, 207, 290-291. 